\title{
3-Dimensional reconstruction of fluorescent structures in tardigrades
}

\author{
Martin PFANNKUCHEN, Franz BRÜMMER and Ralph O. SCHILL* \\ Universität Stuttgart, Biological Institute, Department of Zoology, Pfaffenwaldring 57, 70569 Stuttgart, Germany \\ *e-mail corresponding author: ralph.schill@bio.uni-stuttgart.de
}

\begin{abstract}
Tardigrades are microscopic animals, thus brightfield microscopy is a well established method for tardigrade observation. Modern techniques in functional genetics like fluorescence in situ hybridisation or fluorescently labelled expression markers demand high resolution fluorescence microscopy. Nevertheless tardigrades are still considered to be difficult objects for fluorescence techniques as they are covered by an opaque and diffracting cuticle. We show a modern technique of structured light illumination that enables us to acquire thin optical sections and consequently to reconstruct 3-dimensional structures in tardigrades with a high spatial resolution in all 3 dimensions. This technique is evaluated on taxonomically valuable internal as well as external structures of eutardigrades: the bucco-pharyngeal apparatus and the claws. The 3-dimensional reconstructions allow the measurement of distances in all 3 dimensions.
\end{abstract}

Keywords: structured illumination, optical sectioning, fluorescence microscopy, ApoTome, 3-dimensional rendering, Tardigrada, measuring $3 D$ structures

\section{INTRODUCTION}

Tardigrades are microscopic small animals within their own phylum consisting of more than 960 species (Nelson 2001; Maas \& Waloszek 2001; Guidetti \& Bertolani 2005). The first occurrence of tardigrades is reported from the mid Cambrian (Müller et al. 1995). Eutardigrades have been present at least for 90 million years (Bertolani \& Grimaldi 2000; Budd 2001; Jørgensen \& Kristensen 2004). Several semiterrestrial species are able to survive extremely adverse conditions like freezing and drying by undergoing cryptobiosis (Bertolani et al. 2004). These extraordinary capacities make tardigrades an interesting phylum for the study of extreme physiologies. Therefore the possibility to localize fluorescently labelled probes or expression markers is of highest interest for the applicability of modern techniques in functional genetics and expression analysis.

The animals bear a chitinous cuticle and their taxonomy mainly refers to their claws, the bucco-pharyngeal apparatus, the cuticle's structure as well as to appendages of the cuticle (Thulin 1928; Ramazzotti 1962; Pilato 1969, 1975); Guidetti \& Bertolani 2005). Whilst all these characters are examined microscopically, and brightfield illumination techniques are well established for tardigrade examination, tardigrades are still said to be difficult objects for fluorescence microscopy (Russell et al. 2001). This is mainly due to the opaque and diffracting properties of the cuticle (Guidetti et al. 2000). Apart from dye penetration problems, in epifluorescence microscopy and especially in laser scanning microscopy unpredictable diffraction of illumination and emitted light is a major problem (Russell et al.
2001). While many modern techniques, especially in functional genetics (fluorochrome-coupled proteins, fluorescence in situ hybridisation (fish), antibodystaining), demand high resolution detection of fluorescent signals, the spatial distribution of these signals gives additional and valuable information about their functions. To overcome the aforementioned obstacles physical sectioning or just cutting to get rid of the diffracting cuticle and to facilitate dye penetration are proposed (Russell et al. 2001).

As a first step towards fluorescence microscopy in tardigrades with a high spatial resolution, we decided to reconstruct the 3-dimensional (3D) structure of taxonomically valuable, autofluorescent morphological characters: the bucco-pharyngeal apparatus and the claws.

For fluorescence microscopy we employed the ApoTome to acquire optical sections by application of a so-called structured illumination (Neil et al. 1997). This wide field illumination fluorescence technique proved itself to be applicable in tardigrades and not very susceptible to diffraction of the cuticle. In addition it resulted in a good resolution of the observed structures. Therefore we preferred the structured wide-field illumination (Neil et al. 1998). The 3D reconstructions enable us to measure taxonomically important structures and give us a good idea of their morphology.

\section{METHODS}

Tardigrade specimens of the species Milnesium tardigradum Doyère, 1840 and Macrobiotus tonollii Ramazzotti, 1956 were cultured on agar plates for a couple of years, and subsequently used for the 3D reconstruction of fluorescent structures. Originally they 


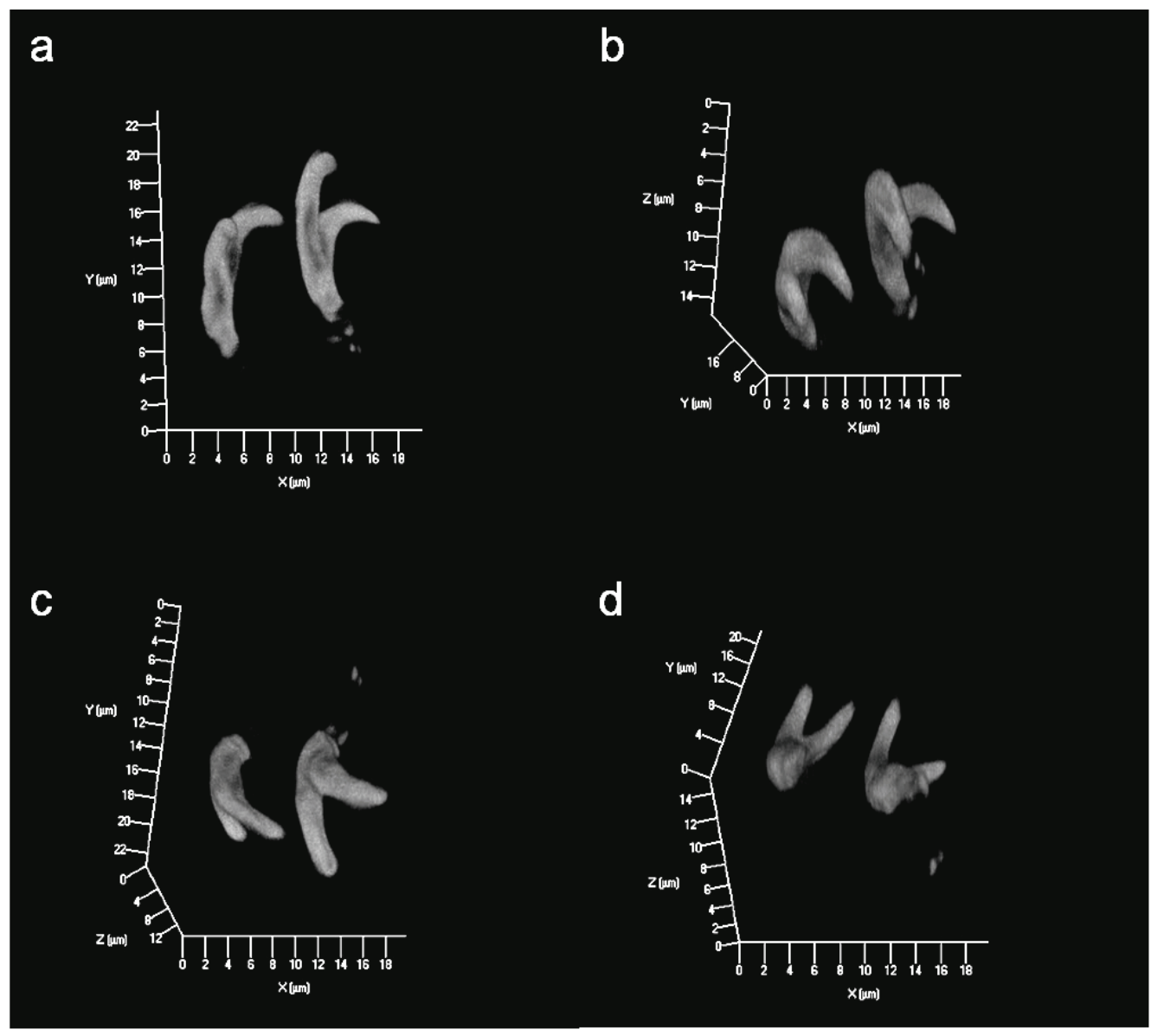

Fig. 1. Macrobiotus tonollii. a-d: Rotation around the $\mathrm{x}$-axis and consequently different aspects of the claws on the right front leg. During rotation of the 3-dimensional reconstruction all details of the structure become visible and thus even initially obstructed parts become accessible.

were collected from moss (covering rock) in Tübingen, Germany and Eugene, Oregon, U.S.A. respectively. The carnivorous tardigrades were reared in petri dishes on a thin layer of $3 \%$ agar. A layer of $3 \mathrm{~mm}$ Volvic ${ }^{\mathrm{TM}}$ water was added. For food, rotifers of the species Philodina citrina Ehrenberg, 1832 were provided twice a week, which were cultured separately in Volvic ${ }^{\mathrm{TM}}$ water and fed with the green algae Chlorogonium sp. All cultures were kept in a climate chamber at $20^{\circ} \mathrm{C}$.

Without previous fixation single specimens were embedded directly in polyvinyl-lactophenol on a $1 \mathrm{~mm}$ object carrier under a $0.13 \mathrm{~mm}$ cover-glass. To check whether the applied method and the necessary autofluorescence is generally valid, 15 specimens of each species were examined. All microscopy was performed on an Axiovert $200 \mathrm{M}$ (Zeiss, Oberkochen, Germany), equipped with an ApoTome unit and a PlanApochromat $63 \times$ objective. Stacks of optical sections were acquired with an Axiocam MRm using the ApoTome with the high magnification grid. Exposuretime was $100 \mathrm{~ms}$. For each stack, according to the dimensions of the structure, 40 to 60 optical sections with a distance of $0.275 \mu \mathrm{m}$ were acquired. Each section was averaged 3 times and the channels for filter sets 10 and 43 were adjusted to avoid chromatic z-axis aberrations. Those stacks were acquired and processed using the Axiovision 4.5 software package. Filter sets used were: Filter set 10 BP450-490|BS510|BP515-565 (drawn in green) and filter set 43 BP550/25|BS570|BP605/70.

\section{RESULTS}

After illumination with blue and green light we could detect autofluorescent emission of green and red light respectively. This autofluorescence was significantly high in the bucco-pharyngeal apparatuses and the claws of Macrobiotus tonollii and in the claws of Milnesium tardigradum. The resulting fluorescence intensity was easily detectable against the diffuse background signal of the cuticle and the tardigrades' cells. That way, structured wide-field illumination applying the ApoTome resulted in thin optical sections. Stacks of these optical sections were processed resulting in 3D reconstructions of these autofluorescent structures. To give an idea of the easy handling of 3D reconstructions, figure 1 shows 4 different aspects of the claws on the right foreleg of $M$. tonollii. By turning the 3D reconstruction of these claws every detail of 

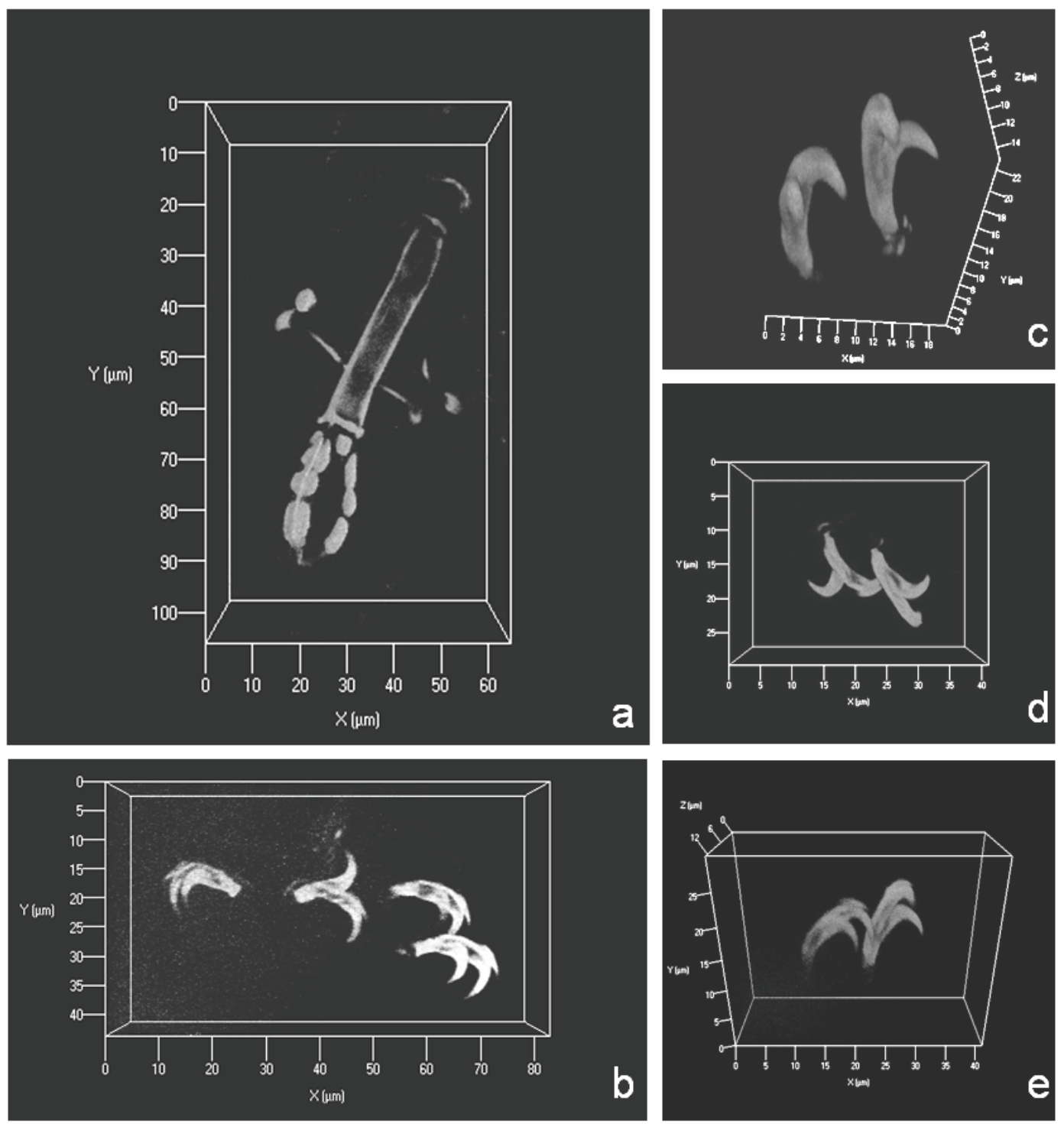

Fig. 2. Macrobiotus tonollii. a: 3-dimensional (3D) reconstruction of the bucco-pharyngeal apparatus after combination of the signals acquired with the filter sets 10 and 43 . b: $3 \mathrm{D}$ reconstruction of the claws on the hind legs (left and right) (filter set 43). c: 3D reconstruction of the right front claws (filter set 43). d: 3D reconstruction of the claws on the second leg (right) (filter set 43). e: 3D reconstruction of the claws on the third leg (right) (filter set 43).

their morphology can be shown and measured, even when initially being obstructed by parts of overlying objects. Figure $2 \mathrm{a}$ displays the $3 \mathrm{D}$ reconstruction of the bucco-pharyngeal apparatus of $M$. tonollii as an overlay of green and red autofluorescence (filter sets 10 and 43 respectively). The placoids are clearly resolved, showing that the first two placoids are joined. In opposition to the buccal-tube and the placoidal structures the stylets are of different material and thus do not emit any autofluorescence. Parts of the buccal tube as well as parts of the mouth also show only weak or no fluorescence, again hinting to differences in the material. Figure $2 \mathrm{~b}$ shows the claws on the right hind leg of M. tonollii, which is shown in different aspect angles in figure 1a-d. Figures 2c-e show 3D reconstructions of the claws on the first, second and third legs of $M$. tonollii. All claw structures show a strong autofluorescence.

Microscopy of specimens 5 weeks after embedding showed no significant fading of the autofluorescence signal (not shown) and no change in morphology as well.

The software Axiovision allows the measurement of distances in $3 \mathrm{D}$ reconstructions. Thus measurements of initially obstructed areas which are obtained from different focal layers are now possible. Figure 3 demonstrates the accuracy and possibilities of these measurements. Figure 3a shows a claw on the third leg of $M$. tardigradum displayed in differential interference contrast (DIC). The claw measures $18.76 \mu \mathrm{m}$ from the base 

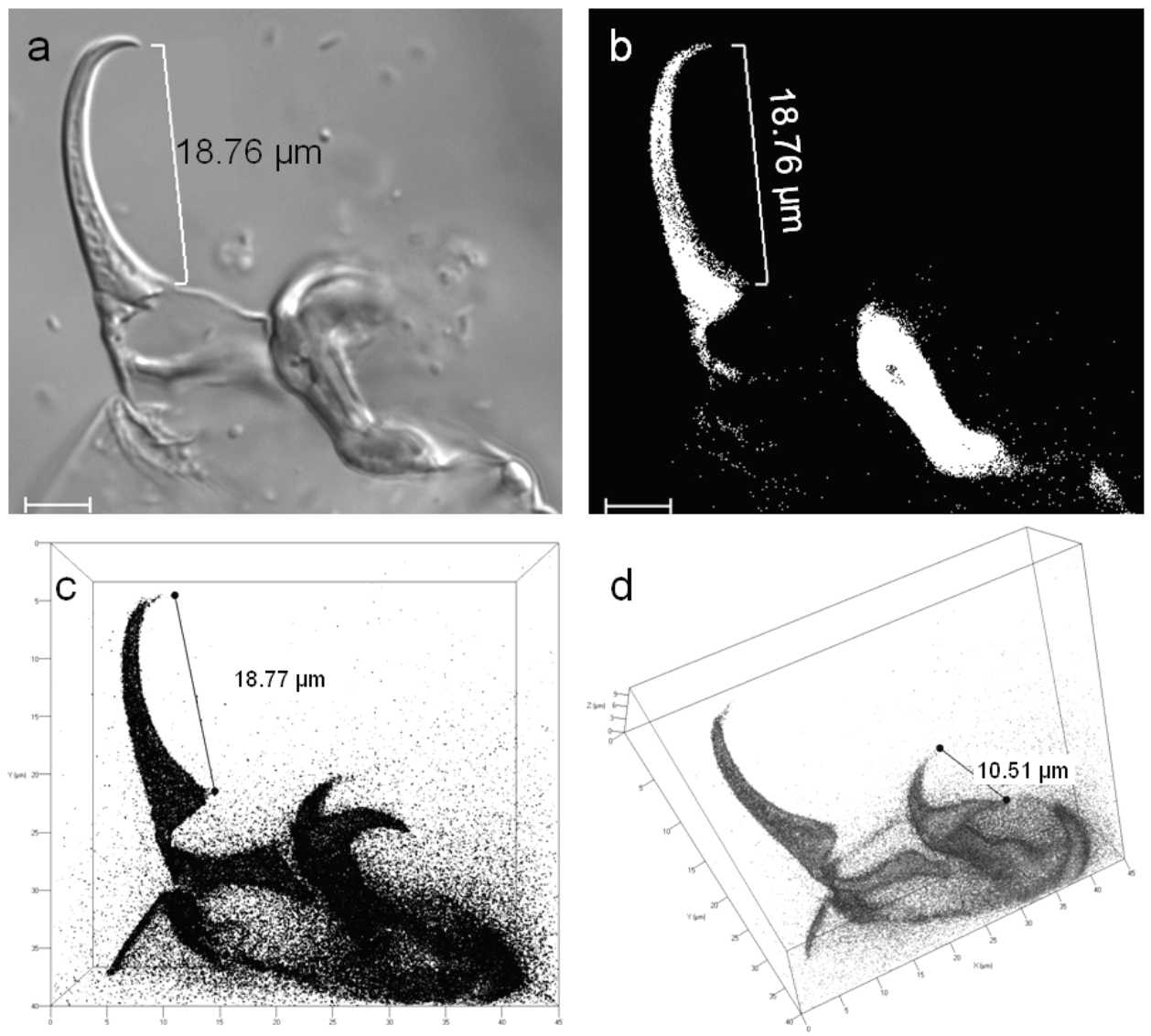

Fig. 3. Milnesium tardigradum. a: Light micrograph (DIC) of the claws on the third leg. b: Fluorescence of the claws on the third leg (filter set 43), 2-dimensional representation. c: 3-dimensional (3D) reconstruction of the autofluorescent claws on the third leg (filter set 43). d: 3D reconstruction of the claws on the third leg (filter set 43) from different aspect angle. a-c show a measurement of the same distance in 2-dimensional as well as 3D representation. $\mathrm{d}$ shows the measurement of the distance between the two tips of the smaller claw, which originally obstructed each other, lying in different focal planes. After turning the 3D reconstruction both tips became visible and thus measurable. Scale bar: $a, b=5 \mu \mathrm{m}$.

to the tip. As it is oriented in the focal layer it is assumed to be the correct value. The same value, of course, is obtained when measuring the same claw's 2D fluorescence image (filter set 43; Fig. 3b). Figure $3 \mathrm{c}$ shows the $3 \mathrm{D}$ reconstruction of the claw (filter set 43 ) and the corresponding measurement, giving a distance of $18.77 \mu \mathrm{m}$. Figure $3 \mathrm{~d}$ shows a different aspect of the reconstruction allowing the measurement of the distance of $10.51 \mu \mathrm{m}$ between the two tips of the smaller claw, which were originally situated in different focal layers (Fig. 3a) and thus their distance is only measurable after $3 \mathrm{D}$ reconstruction.

\section{DISCUSSION}

The claws and bucco-pharyngeal apparatuses of eutardigrades are, among others, important taxonomic structures (Ramazzotti \& Maucci 1983; Guidetti \& Bertolani 2005) that show convenient autofluorescence in green and red light after excitation with blue and green light respectively. The autofluorescence intensity is significantly high, enabling its clear detection above the diffuse background autofluorescence of the tardigrade's body. After careful adjustment of the fluorescence filter sets, exposure time, contrast, brightness, stack number and stack distance, after correction of the chromatic aberrations and after choosing the correct thresholds, a considerably high resolution in all 3 dimensions can be achieved, enabling the correct rendering of internal as well as exposed structures of tardigrades. The resulting 3D reconstructions of these structures are not only convenient visualizations but enable measurements of exact distances within these structures with a putative taxonomic value. The claws could be reconstructed irrespective of their position. Even when lying underneath the specimen, with light having to penetrate the tardigrade twice, the resulting optical sections resulted in good quality reconstructions. The bucco-pharyngeal apparatus represents an internal structure of eutardigrades, surrounded by the chitinous, opaque cuticle. Even these internal structures could be reconstructed with high spatial resolution. Both the claws and the bucco-pharyngeal apparatus are still in place, fixed on the whole specimen, thus showing their 
natural position representing the in situ habitus of the tardigrade.

After the reconstruction of the 3D structure all distances in the object are measurable irrespective of the object's initial orientation, possible obstructions or different focal layers. The measurements are almost identical to the values obtained from classical light micrographs. The difference of $0.05 \%$ is most probably due to a slightly different choice for the beginning and ending of the distance, thus an error introduced by the experimenter. Keeping this and the value of $0.05 \%$ in mind, we consider the measurement within the reconstructions as accurate. Accurate measurements of distances as well as angles within the aforementioned structures might prove valuable in future descriptions and discussions related to taxonomy, ecology and probably other topics concerning tardigrades. However their significance will be subject to further investigations.

Autofluorescence intensities of the claws and the bucco-pharyngeal apparatuses were comparable to or lower than fluorescence in DAPI, FITC, cy3 or cy5 labelled objects. That leads to the conclusion that those aforementioned fluorochromes (which are not the brightest ones available) will work very well in tardigrades, when detected as described here.

Tardigrade specimens are known to change (e.g. degradation of tissue and cuticular structures) with time when embedded in polyvinyl-lactophenol. However no changes were observable within 5 weeks. This might be different after a longer period of time. However embedding in highly viscous material is inevitable because the reduced movement of the object during the acquisition of the focal stacks results in a significantly increased resolution.

\section{CONCLUSIONS}

The method of structured illumination to obtain optical sections applying the Zeiss ApoTome is easily applied, works on specimens embedded already weeks before and requires only one single specimen to be embedded. All characters of taxonomic importance can be revealed on this single specimen irrespective of their position. That way the method is certainly valuable for taxonomic work and results in didactically valuable 3D models of taxonomically important characters (Miller 2004; Guidetti \& Bertolani 2005). In comparison or opposition to scanning electron microscopy the preparation for the described method is less laborious, thus faster, and this light-microscopical method produces 3D reconstructions that also reveal their covered side "underneath" because they can be turned around after reconstruction (Eibye-Jacobson 2001). Due to the whole specimen being embedded, the claws and bucco-pharyngeal apparatuses are considered to be still in place and thus the reconstructions should represent the in situ situation without preparation artefacts. All morphological characters are resolved by the described technique.
Measurements of distances in all 3 dimensions are shown to be possible and accurate as well as easily accessible from one single specimen. Those measurements and their significance in taxonomy, ecology and further topics related with tardigrades will be subject to further investigations.

As even internal structures, like the bucco-pharyngeal apparatus, with an autofluorescence intensity comparable to the fluorescence intensities of well established fluorochromes, are easily detectable, we consider the tardigrades ready for further investigations including fluorescence detection of fluorochromes with a high spatial resolution referring to the life-habitus of the specimen.

\section{ACKNOWLEDGMENTS}

We wish to thank the editors Lorena Rebecchi and Giovanni Pilato for the helpful advice and critical discussion on the manuscript. We are also grateful to the anonymous reviewers for constructive suggestions. This study was supported by the German Research Foundation (DFG), SCHI865/1-1, and enabled using the equipments made available by the project BIOTECmarin (03F0414D) funded by the German Federal Ministry of Education and Research and the Universität Stuttgart.

\section{REFERENCES}

Bertolani, R. \& D. Grimaldi. 2000. A new eutardigrade (Tardigrada: Milnesiidae) in amber from the Upper Cretaceous (Turonian) of New Jersey. In: D. Grimaldi (Ed.), Studies on fossils in amber, with particular reference to the Cretaceous of New Jersey. Leiden, The Netherlands, Backhuys Publishers: 103-110.

Bertolani, R., R. Guidetti, K.I. Jönsson, T. Altiero, D. Boschini, L. Rebecchi. 2004. Experiences with dormancy in tardigrades. J. Limnol., 63 (Suppl. 1): 16-25.

Budd, G.E. 2001. Tardigrades as "Stem-Group Arthropods": The Evidence from the Cambrian Fauna. Zool. Anz., 240: 265-279.

Eibye-Jacobson, J. 2001. A new method for making SEM preparations of the tardigrade buccopharyngeal apparatus. Zool. Anz., 240: 309-319.

Guidetti, R. \& R. Bertolani. 2005. Tardigrade taxonomy: an updated check list of the taxa and a list of characters for their identification. Zootaxa, 845: 1-46.

Guidetti, R., L. Rebecchi \& R. Bertolani. 2000. Cuticle structure and systematics of the Macrobiotidae (Tardigrada, Eutardigrada). Acta Zool., 81: 27-36.

Jørgensen, A. \& R.M. Kristensen. 2004. Molecular phylogeny of Tardigrada - investigation of the monophyly of Heterotardigrada. Mol. Phylogenet. Evol., 32: 666-670.

Maas, A. \& D. Waloszek. 2001. Cambrian Derivatives of the Early Arthropod Stem Lineage, Pentastomids, Tardigrades and Lobopodians An 'Orsten' Perspective. Zool. Anz., 240: 451-459.

Miller, W.R. 2004. Tardigrades as a teaching and research tool. J. Pennsylvania Acad. Sci., 77: 142-143.

Müller, K.J., D. Walossek \& A. Zakharov. 1995. 'Orsten' type phosphatized soft-integument preservation and a new record from the Middle Cambrian Kuonamka Formation in Siberia. N. Jahrb. Geol. Palaeontol. Abh., 197: 101-118. 
Neil, M.A.A., R. Juskaitis \& T. Wilson 1997. Method of obtaining optical sectioning by using structured light in a conventional microscope. Opt. Lett., 22: 1905-1907.

Neil, M.A.A., T. Wilson \& R. Juskaitis. 1998. A light efficient optically sectioning microscope. J. Microsc., 189: 114-17.

Nelson, D.R. 2001. Tardigrada. In: J.H. Thorp \& A.P. Covich (Eds), Ecology and Classification of North American Freshwater Invertebrates. 2nd Edition. San Diego, California, USA; London, England, UK, Academic Press, Inc: 527.

Pilato, G. 1969. Evoluzione e nuova sistemazione degli Eutardigradi. Boll. Zoll., 36: 327-345.
Pilato, G. 1975. On the systematic criteria of the Eutardigrada. Mem. Ist. ital. Idrobiol., 32: 277-303.

Ramazzotti, G. 1962. Il Phylum Tardigrada. Mem. Ist. ital. Idrobiol., 14: 1-595.

Ramazzotti, G. \& W. Maucci. 1983. Il Phylum Tardigrada. Terza Edizione riveduta e aggiornata. Mem. Ist. ital. Idrobiol., 41: 1-1012.

Russell, P.M., N.J. Marley \& M.E. Hockings. 2001. Do confocal microscopy and tardigrades have a future together? Zool. Anz., 240: 543-548.

Thulin, G. 1928. Uber die Phylogenie und das System de Tardigraden. Hereditas, 207-226. 